\title{
Single-sided mobile NMR apparatus using the transverse flux of a single permanent magnet
}

\author{
Wei-Hao Chang ${ }^{\mathrm{a}}$, Jyh-Horng Chen ${ }^{\mathrm{a}}$, Lian-Pin Hwang ${ }^{\mathrm{b}, \mathrm{c}, *}$ \\ ${ }^{a}$ Department of Electrical Engineering, National Taiwan University, Taipei 106, Taiwan \\ ${ }^{\mathrm{b}}$ Department of Chemistry, National Taiwan University, Taipei 106, Taiwan \\ ${ }^{\mathrm{c}}$ Institute of Atomic and Molecular Science, Academia Sinica, Taipei 106, Taiwan \\ Received 27 December 2008; revised 26 April 2009; accepted 10 May 2009
}

\begin{abstract}
This study presents a simple design for a mobile, single-sided nuclear magnetic resonance (NMR) apparatus which uses the magnetic flux parallel to the magnetization direction of a single, disc-shaped permanent magnet polarized in radial direction. The stray magnetic field above the magnet is approximately parallel to the magnetization direction of the magnet and is utilized as the $\mathrm{B}_{0}$ magnetic field of the apparatus. The apparatus weighs $1.8 \mathrm{~kg}$, has a compact structure and can be held in one's palm. The apparatus generates a $\mathrm{B}_{0}$ field strength of about $0.279 \mathrm{~T}$ at the center of apparatus surface and can acquire a clear Hahn echo signal of a pencil eraser block lying on the RF coil in one shot. Moreover, a strong static magnetic field gradient exists in the direction perpendicular to the apparatus surface. The strength of the static magnetic field gradient near the center of the apparatus surface is about $10.2 \mathrm{~T} / \mathrm{m}$; one-dimensional imaging of thin objects and liquid selfdiffusion coefficient measurements can be performed therein. The available spatial resolution of the one-dimensional imaging experiments using a $5 \times 5 \mathrm{~mm}$ horizontal sample area is about $200 \mu \mathrm{m}$. Several nondestructive inspection applications of the apparatus, including distinguishing between polyethylene grains of different densities, characterizing epoxy putties of distinct set times and evaluating the fat content percentages of milk powders, are also demonstrated. Compared with many previously published designs, the proposed design bears a simple structure and generates a $B_{0}$ magnetic field parallel to the apparatus surface, simplifying apparatus construction and simultaneously rendering the selection of the radiofrequency coil relatively flexible.
\end{abstract}

(C) 2009 Elsevier Inc. All rights reserved.

Keywords: Unilateral magnetic resonance; One-dimensional imaging; Spatial resolution; Diffusion; Non-destructive inspection

\section{Introduction}

A single-sided nuclear magnetic resonance (NMR) apparatus [1-21] can acquire an NMR signal of an experimental object located outside the apparatus; the size of the experimental object is not limited by the dimensions of the device. For ordinary NMR equipment, the object under investigation is encompassed by the magnet structure and; therefore, the size of the object is restricted by the dimensions of the bore or gap of the magnet. However, for a single-sided NMR apparatus, the static magnetic field $\mathrm{B}_{0}$

\footnotetext{
* Corresponding author. Department of Chemistry, National Taiwan University, Taipei, 106 Taiwan. Tel.: +8862 33661166; fax: +886233663294.

E-mail address: nmrb@pub.iams.sinica.edu.tw (L.-P. Hwang).
}

and radiofrequency $(\mathrm{RF})$ magnetic field $\mathrm{B}_{1}$ are located outside the magnet; the $\mathrm{B}_{0}$ and $\mathrm{B}_{1}$ magnetic fields are perpendicular to each other in some space outside the magnet, where the sensitive volume of the single-sided NMR apparatus is defined. When the resonance condition $\omega_{0}=\gamma \mathrm{B}_{0}$ is satisfied in the sensitive volume, the nuclei of the experimental object located in the sensitive volume are excited by the $B_{1}$ magnetic field. The NMR signal of the object is then detected by the RF coil. In this configuration, the magnet does not need to surround the experimental object; hence, no restriction exists for the size of the experimental object. The ability to noninvasively inspect an experimental object of any size renders the single-sided NMR apparatus suitable for polymer quality control [22-24] and culture heritage evaluation [25-28], etc. Various methods and applications of the single-sided NMR apparatus are comprehensively described in Ref. [3]. 
This work presents a simple and workable design for a single-sided NMR apparatus. The proposed scheme mainly consists of a single, disc-shaped permanent magnet polarized in radial direction and a planar RF coil positioned slightly above the magnet. Fig. 1A presents a simplified illustration of the design. Intuitively, the stray magnetic field generated by the magnet will surround the magnet. Due to the spatial symmetry of the distribution of magnetization of the magnet, the direction of the stray magnetic field in the space above the magnet will be approximately parallel to the magnetization direction of the magnet and the magnet surface; this direction is defined as the $z$ direction (Fig. 1A). In this article, the $x$ direction is defined also in the plane parallel to the magnet surface but is perpendicular to the $z$ direction; the $y$ direction is normal to the magnet surface (Fig. 1A). A planar RF coil producing a $\mathrm{B}_{1}$ magnetic field in the $x$ or $y$ directions can be positioned slightly above the magnet, and an experimental object placed above the RF coil can thus be investigated via NMR methods. Many published designs employ two or several permanent magnet blocks instead of only one magnet block to constitute the apparatus and

A

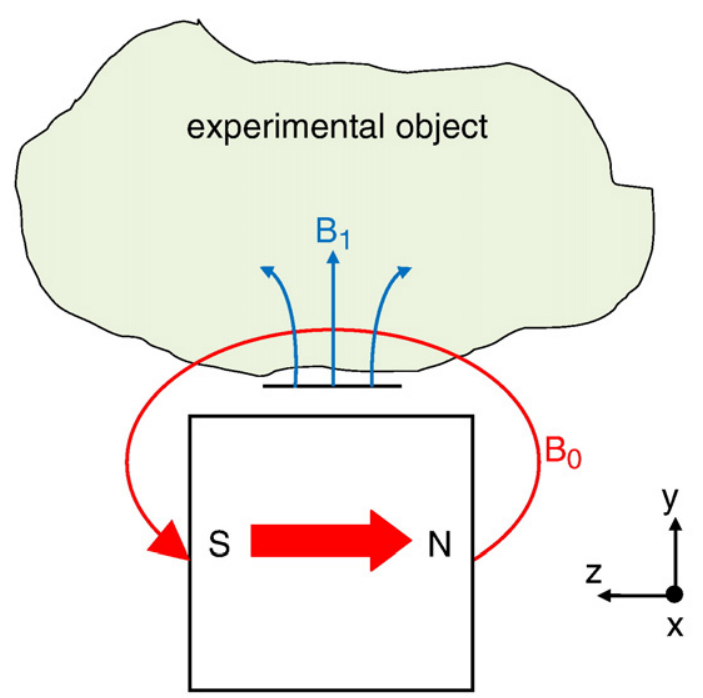

B

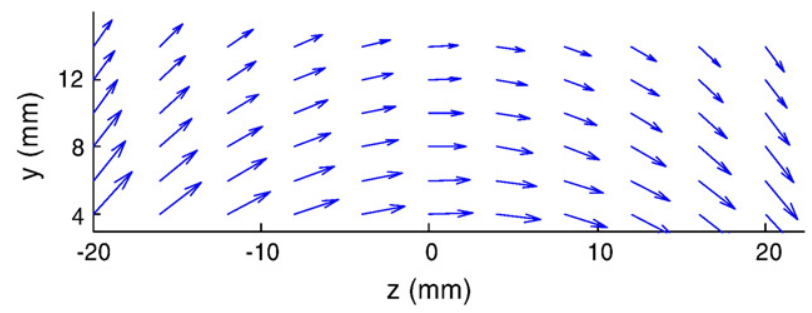

Fig. 1. (A) A schematic drawing of the proposed single-sided NMR apparatus using a single, disc-shaped permanent magnet polarized in radial direction. The horizontal black line slightly above the magnet represents the planar RF coil; the thick arrow inside the magnet represents the magnetization direction of the magnet. (B) The measured directions of the $\mathrm{B}_{0}$ magnetic field in the $\mathrm{yz}$ plane at $x=0 \mathrm{~mm}$. The arrow length is proportional to the $\mathrm{B}_{0}$ magnetic field strength. achieve some desirable performance such as uniform static gradient strength or good $\mathrm{B}_{0}$ homogeneity. However, the magnet blocks usually have manufacturing tolerances in their remanence, which possibly degrades the performance of the built apparatus. Correcting this problem by preparing permanent magnet blocks with nearly identical remanence in advance or by adding shim magnets or pole pieces often requires additional cost or effort. Additionally, handling and fixing a group of magnet blocks may cause safety concern and usually needs extra care. In some applications that only general parameters such as the relaxation times of an experimental object are measured, using the proposed onemagnet design is sufficient and can reduce the effort and cost for apparatus construction. Moreover, the simple shape of the magnet also makes fabrication of the magnet relatively easy. Although our design and the previously published, axiallymagnetized single bar magnet design of Blümich et al. [5] are quite similar and only differ in the magnetization direction, our design results in a $\mathrm{B}_{0}$ magnetic field parallel to the apparatus surface and consequently allows the apparatus to use a simple loop RF coil $[3,10]$ or an RF coil of the figureeight type $[3,5,29]$. The selection the RF coil is therefore relatively flexible $[10,17,19]$, which may be helpful to fit the apparatus for different experimental conditions, such as different detection depths or lateral selection areas $[3,10]$.

A single-sided mobile NMR apparatus was built according to the proposed design. The constructed apparatus has a top area of $14.5 \times 10 \mathrm{~cm}$, height of $8 \mathrm{~cm}$ and weight of $1.8 \mathrm{~kg}$. The $\mathrm{B}_{0}$ magnetic field strength at the center of the apparatus surface is about $0.279 \mathrm{~T}$. The apparatus can acquire a clear Hahn echo of a pencil eraser block placed directly on the planar RF coil in one shot. Moreover, the apparatus generates a strong static magnetic field gradient $\Delta \mathrm{B}_{0} / \Delta_{\mathrm{y}}$ normal to the apparatus surface. The static gradient near the center of the apparatus surface is relatively uniform and has a strength of about $10.2 \mathrm{~T} / \mathrm{m}$, enabling the apparatus to perform onedimensional imaging of the thin object with a submillimeter thickness and measure the self-diffusion coefficients of liquids. Several nondestructive inspection experiments were also conducted by the proposed apparatus, including distinguishing between polyethylene grains of different densities by measuring the spin-spin $\left(\mathrm{T}_{2}\right)$ relaxation decays, characterizing the hardening process of different epoxy putties by continuously measuring the effective $\mathrm{T}_{2}$ relaxation times using the Carr-Purcell-Meiboom-Gill (CPMG) pulse sequence, and correlating fat content percentages of mixed milk powders with their CPMG echo amplitudes.

\section{Apparatus construction}

A disc-shaped neodymium-iron-boron permanent magnet polarized in radial direction (diameter: $6 \mathrm{~cm}$; height: $5 \mathrm{~cm}$ ) was purchased from Ming Yen Electronics (Taichung, Taiwan). The stray magnetic field above the magnet was utilized as the $\mathrm{B}_{0}$ magnetic field. Before hardware imple- 
mentation, design feasibility was evaluated and verified using the commercial simulation software package Maxwell3D (Ansoft, Pittsburgh, PA, USA). Actually, Maxell3D simulations indicate that magnets of other shapes (e.g., a cuboid magnet) also can be used. A gaussmeter (Model 7010, F. W. Bell, Orlando, FL, USA) with a transverse-type Hall probe was used to find the poles and magnetization direction of the magnet. By mounting the Hall probe on a three-axis translation stage and adjusting the orientation of the Hall probe, three orthogonal components of the $\mathrm{B}_{0}$ magnetic field above the magnet were scanned with a spatial resolution of $1 \mathrm{~mm}$. The measurement origin was defined at the center of the top surface of the magnet. As expected, the measurement result indicates that the direction of the $\mathrm{B}_{0}$ magnetic field above the center of the magnet surface is parallel to the magnetization direction of the magnet and the magnet surface (Fig. 1B).

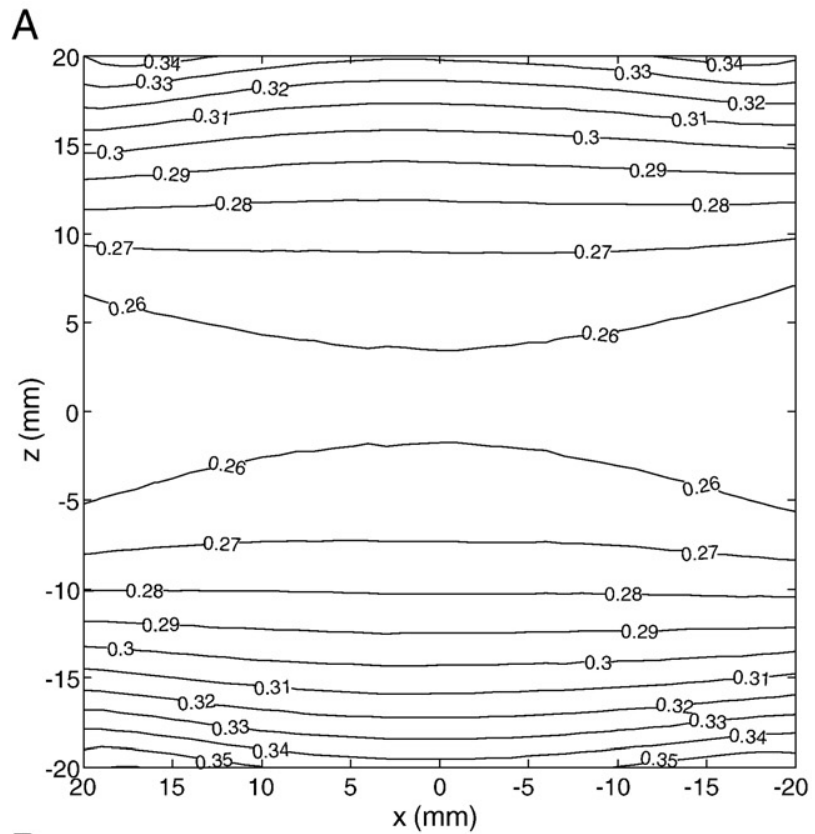

B
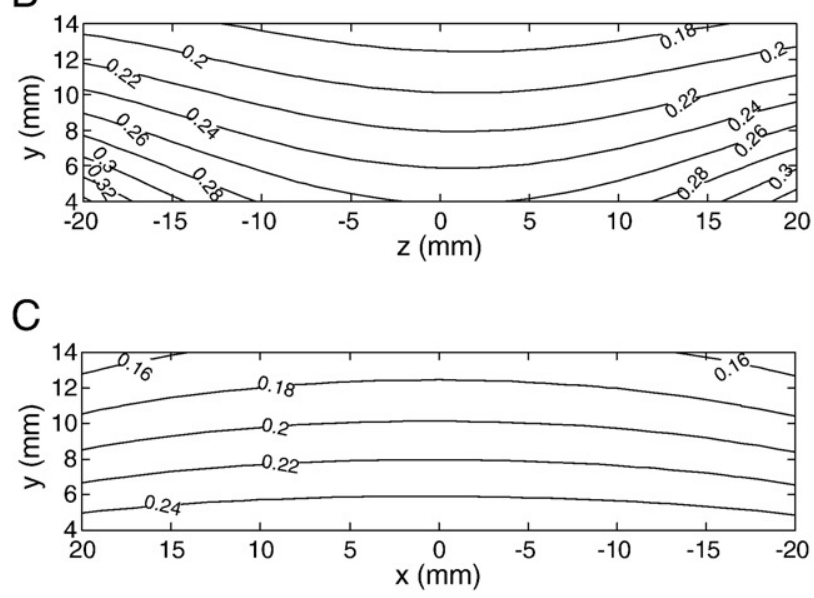

Because the sensing point of the Hall probe is 1.5 in. (about $3.8 \mathrm{~mm}$ ) away from the probe tip, and this distance hinders field measurement in the space proximal to the magnet surface, the $\mathrm{B}_{0}$ magnetic field was measured above $y=4 \mathrm{~mm}$. Maxwell3D simulations were conducted to estimate the profile of the $\mathrm{B}_{0}$ magnetic field below $y=4$ $\mathrm{mm}$; the simulation result was manually scaled such that it could fit the measurement result above $y=4 \mathrm{~mm}$ as closely as possible. Fig. $2 \mathrm{~A}-\mathrm{C}$ display the measured contour plots of the $\mathrm{B}_{0}$ magnetic field strength. Fig. $2 \mathrm{~B}$ indicates that the measured $\mathrm{B}_{0}$ contour lines have a small offset (about $1 \mathrm{~mm}$ ) in the $z$ direction, which is likely caused by the manufacturing tolerances of the purchased magnet. Fig. 2D shows the measured and simulated $\mathrm{B}_{0}$ strength along the $y$-axis. Due to the small differences between the simulation and measurement results above $y=4 \mathrm{~mm}$, predicting the profile of the $\mathrm{B}_{0}$ magnetic field below $y=4 \mathrm{~mm}$ using the simulation result is
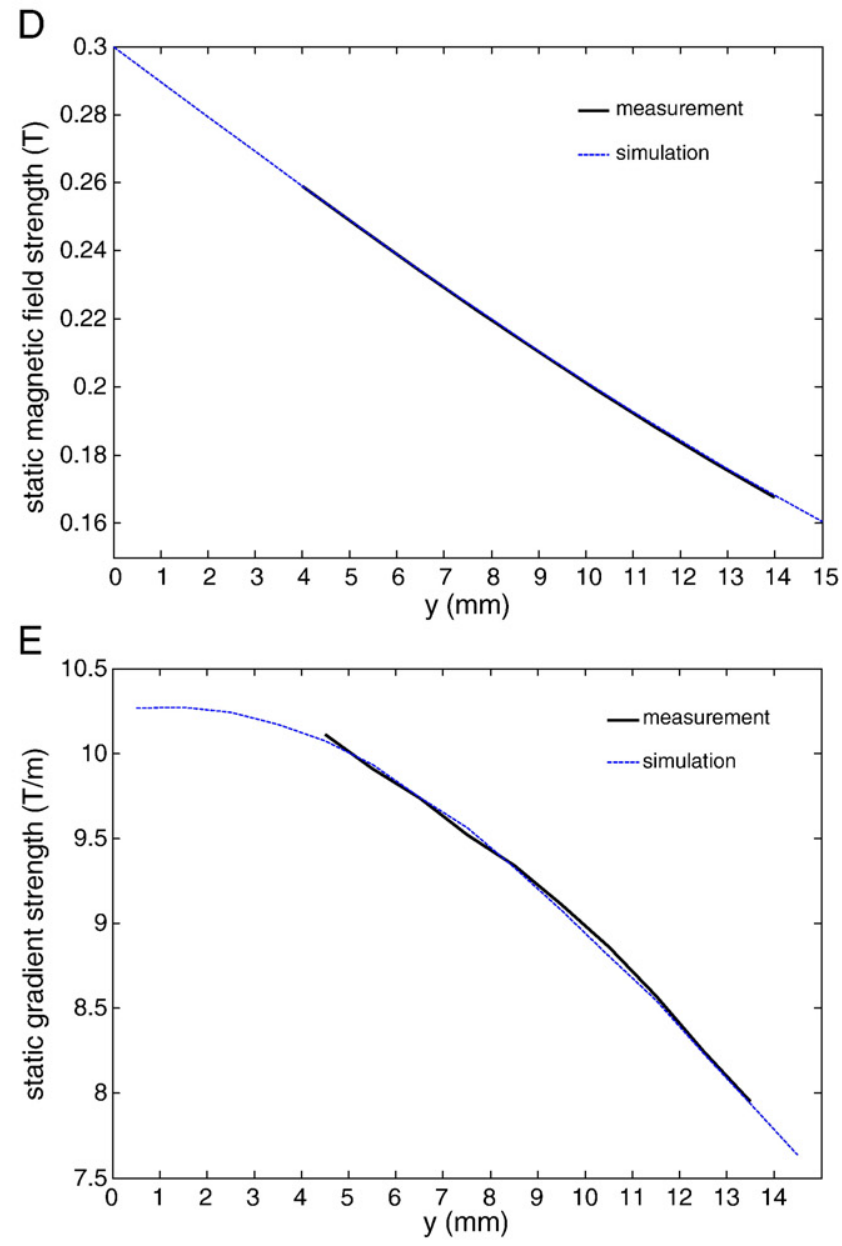

Fig. 2. Measured contour plots of the $\mathrm{B}_{0}$ magnetic field strength (A) in the xz plane at $y=4 \mathrm{~mm},(\mathrm{~B})$ in the yz plane at $x=0 \mathrm{~mm}$, and (C) in the $\mathrm{xy}$ plane at $z=0 \mathrm{~mm}$. (D) Measured and simulated $\mathrm{B}_{0}$ strength along the $\mathrm{y}$ axis. (E) Measured and simulated static gradient strength $\left|\Delta \mathrm{B}_{0} / \Delta y\right|$ along the $y$ axis. 
therefore reasonable. The simulation result reveals that the shapes of the $B_{0}$ contour lines within $0 \mathrm{~mm}<y<4 \mathrm{~mm}$ generally resemble those above $y=4 \mathrm{~mm}$, except that curvatures of the contour lines are somewhat different (results not shown). Fig. 2B-D reveal that the $\mathrm{B}_{0}$ magnetic field strength is strongest near the magnet surface and decays with vertical distance from the magnet. Consequently, an experimental object can be placed close to the magnet surface to increase the NMR signal amplitude.

Fig. 2A indicates that in the horizontal (xz) plane, an area with relatively homogeneous $B_{0}$ field strength exists above the center of the magnet surface; this area has a larger dimension in the $x$ direction than that in the $\mathrm{z}$ direction. Consequently, the planar RF coil should only cover this area to achieve efficient RF excitation and signal detection. Fig. $2 \mathrm{~B}$ and $\mathrm{C}$ reveal that a large static magnetic field gradient $\Delta \mathrm{B}_{0} / \Delta y$ exists above the magnet; the vertical distances between adjacent $\mathrm{B}_{0}$ strength contour lines indicate that the static gradient strength $\left|\Delta \mathrm{B}_{0} / \Delta_{\mathrm{y}}\right|$ varies with the spatial position. At the same height, the static gradient strength is larger at peripheral locations than around the central axis of the magnet (the $y$-axis). The flat $\mathrm{B}_{0}$ strength contour lines above the center of the magnet surface suggest that the static gradient strength therein is relatively homogeneous in both the $z$ and $x$ directions. Fig. 2E shows the measured and simulated static gradient strength $\left|\Delta \mathrm{B}_{0} / \Delta_{\mathrm{y}}\right|$ along the $y$-axis and indicates that the static gradient strength is approximately $10.27 \mathrm{~T} / \mathrm{m}$ within $0.5 \mathrm{~mm}<y<1.5 \mathrm{~mm}$; the static gradient strength then gradually decreases as the vertical distance from the surface of the magnet increases. Nevertheless, the largest deviation in the static gradient strength within $0.5 \mathrm{~mm}<y<4.5 \mathrm{~mm}$ remains less than $2 \%$. This static gradient facilitates one-dimensional imaging in the $y$ direction in the space right above the center of the magnet surface, which is shown in Section 3.2.

A $3 \times 1 \mathrm{~cm}$ rectangular $\mathrm{RF}$ coil (Fig. $3 \mathrm{~A}$ ) fabricated on a 0.8-mm-thick printed circuit board (PCB) (Kinsten Electronics, Taipei, Taiwan) was positioned to cover the horizontal area with relatively homogeneous $\mathrm{B}_{0}$ field strength directly above the center of the magnet surface (Fig. 2A). The long side of the RF coil is orientated in the $x$ direction, in which the $\mathrm{B}_{0}$ field homogeneity is better than that in the $z$ direction. Although the proposed design allows the apparatus to use a simple loop RF coil $[3,10]$ that generates a $B_{1}$ magnetic field perpendicular to the coil surface, a pattern of two figure-eight spiral coils connected in series, which is similar to those presented by Blümich et al. [5], was used to improve the signal sensitivity close to the RF coil, where the experimental object will be placed. The counter-wound pattern helps to reduce interference generated by external noise $[3,29]$. Each lobe of the figure-eight spiral coil contains leads of 3.5 turns; the lead width is $0.5 \mathrm{~mm}$ and the lead spacing is $0.25 \mathrm{~mm}$. The $\mathrm{B}_{1 y}$ (in the direction perpendicular to the coil surface) and $\mathrm{B}_{1 x}$ (in the direction linking the spiral centers of the RF coil) components produced by the RF coil were used for RF excitation [29]. The resistance and inductance of the

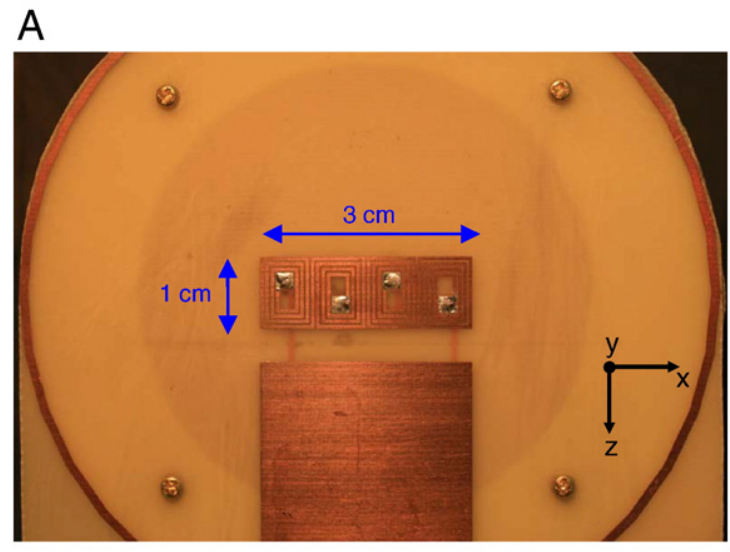

B

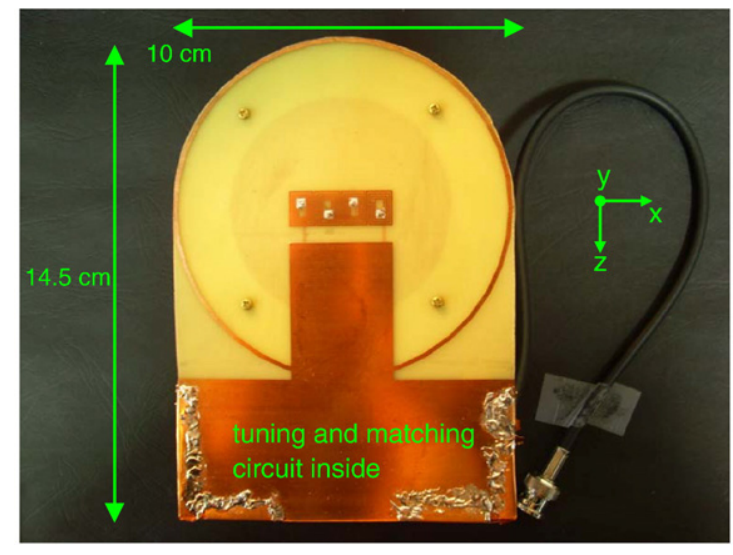

Fig. 3. (A) Top view of the planar RF coil. The lead interconnecting the two sets of figure-eight coils and the leads connecting the RF coil to the tuning circuitry are on the backside of the PCB. (B) Top view of the proposed apparatus. Tuning and matching circuitry is soldered inside the copper box below the RF coil.

RF coil measured using an LCR meter (AX-221; ADEX Corporation, Kyoto, Japan) are $0.46 \Omega$ and $0.5 \mu \mathrm{H}$, respectively. The bottom face of the RF coil was then shielded with a grounded copper layer, which greatly reduces the RF coil ringing caused by the interaction between the RF coil and magnet [29]. The RF coil was tuned by a $270 \mathrm{pF}$ nonmagnetic chip capacitor (American Technical Ceramics, Huntington Station, NY, USA) and a $120 \mathrm{pF}$ nonmagnetic trimmer capacitor (Voltronics Corporation, Denville, NJ, USA) connected in parallel; another $120 \mathrm{pF}$ nonmagnetic trimmer capacitor was used to match the RF coil to an impedance of $50 \Omega$. The tuning and matching capacitors were shielded in a grounded copper box. The magnet was inserted into a polyoxymethylene (POM) plastic holder (inner diameter: $6 \mathrm{~cm}$, outer diameter: $10 \mathrm{~cm}$, height: $8 \mathrm{~cm}$ ); the entire RF coil circuitry was then tightly fixed on the top face of the plastic holder by brass screws. The top face of the RF coil is finally positioned at a height of about $1.9 \mathrm{~mm}$ above the magnet surface. The 3-dB bandwidth of the RF coil circuitry measured at $11.5 \mathrm{MHz}$ by a network analyzer 
(E5061A; Agilent Technologies, Santa Clara, CA, USA) is $0.47 \mathrm{MHz}$, corresponding to a quality factor $Q$ of 24 .

The constructed single-sided mobile NMR apparatus has a top area of $14.5 \times 10 \mathrm{~cm}$, height of $8 \mathrm{~cm}$ and weight of 1.8 $\mathrm{kg}$ (Fig. 3B). The apparatus has a compact structure and can be held in one's palm. To perform NMR experiments, the apparatus was connected to a MARAN DRX spectrometer (Oxford Instruments, Abingdon, Oxfordshire, UK) and a 300-W RF transmitter via a T/R switch (Bruker Corporation, Rheinstetten, Germany). The $\theta_{x}-\tau-2 \theta_{y}-\tau$ - Hahn echo pulse sequence [30] was utilized to collect the NMR signal of an experimental object; the CPMG echo-addition technique $[10,30]$ was often employed to increase the signal-to-noise ratio (SNR). Additionally, the measured $\mathrm{T}_{2}$ relaxation time of the substrate material of the RF coil using the Hahn echo sequence was $36.34 \pm 0.45 \mu$ s. Consequently, an echo time exceeding $200 \mu$ s or a center frequency at least $0.4 \mathrm{MHz}$ less than the resonant frequency at the center of the apparatus surface was used in all experiments. In this manner, the signal from the RF coil substrate was not observed. All experiments were conducted at an ambient temperature of $25 \pm 0.5^{\circ} \mathrm{C}$. Experimental data were processed and plotted using MATLAB (MathWorks, Natick, MA, USA) or ORIGIN (OriginLab Corporation, Northampton, MA, USA) software.

\section{Experimental results}

\subsection{General characteristics of the apparatus}

A small latex sheet $(5 \times 5 \times 0.06 \mathrm{~mm})$ was attached onto the center of the RF coil surface, and CPMG echoes of the latex sheet were acquired to map the $\mathrm{B}_{0}$ magnetic field strength therein. The summed echo displayed in the frequency domain peaked at $11.86 \mathrm{MHz}$ (Fig. 4A, the leftmost peak drawn with a solid line), meaning that the $\mathrm{B}_{0}$ magnetic field strength at the center of the apparatus surface is about 0.279 T. However, if a large latex sheet $(30 \times 10 \times 0.06 \mathrm{~mm})$, which had the same area as the RF coil, was attached onto the RF coil surface and measured in the same way, the acquired echo signal in the frequency domain also peaked at $11.86 \mathrm{MHz}$ (Fig. 4A, the leftmost peak drawn with a dash line), but the base of the peak was much broader than that obtained from the small latex sheet, manifesting the $\mathrm{B}_{0}$ magnetic field inhomogeneity over the entire area of the RF coil. Nevertheless, when an RF pulse of a short length is applied for excitation, which is typically the case for the proposed apparatus, the largest signal sensitivity difference across the RF coil surface measured using the small latex sheet was less than 26\% (Fig. 4B), indicating that each part of the RF coil is effective in signal generation.

Furthermore, the small and the large latex sheets were respectively placed at several vertical positions between 0.45 $\mathrm{mm}$ and $2.85 \mathrm{~mm}$ directly above the RF coil; the $\mathrm{B}_{0}$ field homogeneity at these positions were accessed in the same way (Fig. 4A). The measurement result in Fig. 4A also
A
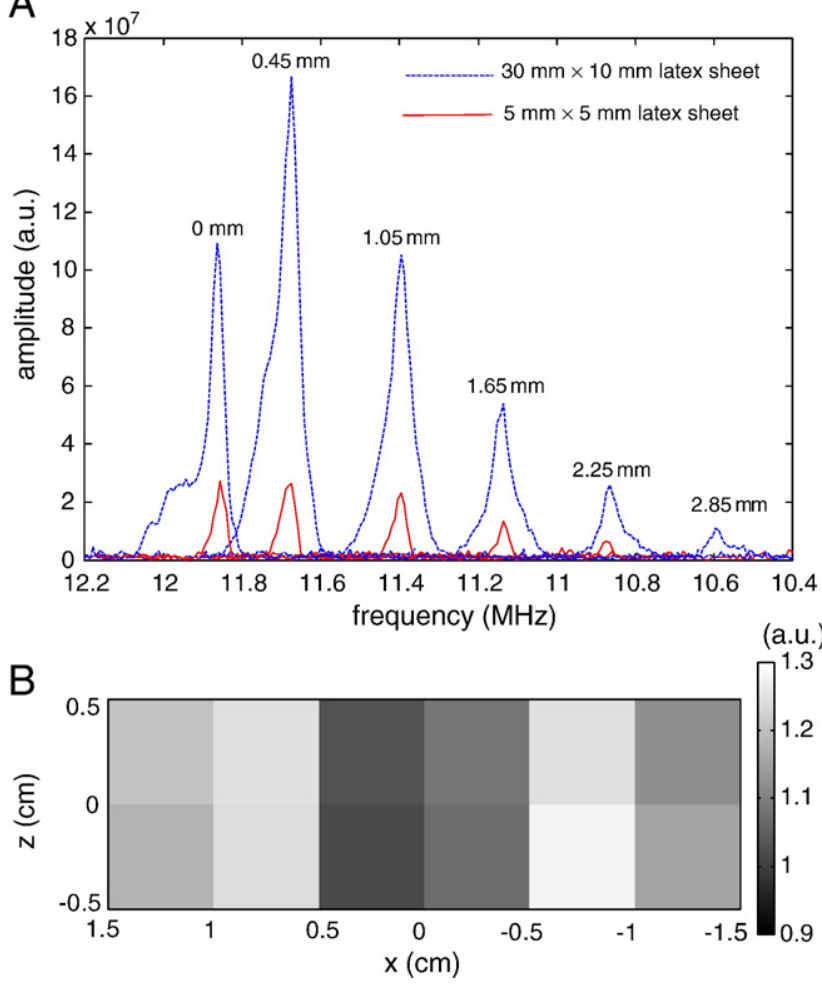

Fig. 4. (A) $B_{0}$ field homogeneity on the RF coil surface or at several different heights directly above the RF coil, measured with the small $(5 \times 5 \times 0.06 \mathrm{~mm})$ and the large $(30 \times 10 \times 0.06 \mathrm{~mm})$ latex sheets. Eight CPMG echoes with an echo spacing of $300 \mu$ s were generated and summed to increase the SNR. During each measurement, the center frequency was adjusted such that the peak of the obtained profile in the frequency domain was exactly on resonance. The values above the profiles denote the vertical distances between the RF coil and the latex sheets. The profile of the small latex sheet at a height of $2.85 \mathrm{~mm}$ above the RF coil was absent because the SNR was too low. Other experimental parameters were RF pulse length of $2 \mu$ s, relaxation delay of $0.1 \mathrm{~s}$ and 3000 averages. For the large latex sheet attached on the RF coil surface (the leftmost peak drawn with a dash line), the nominal $\pi / 2$ pulse is shorter than $2 \mu \mathrm{s}$ used here, leading to signal attenuation and causing the peak amplitude not the biggest one in this figure. (B) The signal sensitivity map of the RF coil obtained by measuring the time-domain summed CPMG echo amplitude of the small latex sheet attached at different areas on the RF coil surface. Experimental parameters were the same as those used in Fig. 4A, except that the center frequency was fixed at $11.86 \mathrm{MHz}$. The signal sensitivity at each location was also modulated by the local pattern of the RF coil.

revealed the slice profiles of the latex sheets at different heights. The profiles of the large latex sheet again displayed broad bases and the profile shape gradually changed as the vertical distance between the RF coil and the latex sheet increased, suggesting different $B_{0}$ field strength distributions in the horizontal plane at different heights (Fig. 4A). In contrast, the profiles of a small latex sheet displayed much narrower bases. Consequently, for one-dimensional imaging in the $y$ direction, an improved spatial resolution can be achieved by restricting the horizontal area of the sample in the region directly above the center of the RF coil; however, this improvement is at the expense of a reduced SNR. 
The sensitivity of the apparatus was obtained by measuring the Hahn echo of a pencil eraser block lying on the RF coil. The maximal echo amplitude of the pencil eraser block occurred at a center frequency of $11.65 \mathrm{MHz}$ with a $2 \mu$ s RF pulse length. In this condition, a clear Hahn echo of the pencil eraser block was acquired in one shot (results not shown). When the detection depth was increased by reducing the center frequency of the RF pulse, the echo amplitude was attenuated considerably. Although using a long RF pulse length increased the echo amplitude at a large detection depth, the resultant echo amplitude remained smaller than that obtained at a small detection depth using a short RF pulse length (Fig. 5), indicating that the most sensitive region of the apparatus is close to the RF coil surface.

\subsection{One-dimensional imaging of layered samples}

The sample for the one-dimensional imaging experiment was composed of small latex sheets $(5 \times 5 \times 0.06 \mathrm{~mm})$; two or four latex sheets were stacked together, and adjacent latex sheets were separated by a 0.2 -mm-thick transparency film. The bottom of the sample was placed $0.45 \mathrm{~mm}$ above the RF coil surface (about $2.35 \mathrm{~mm}$ above the magnet surface), and the sample center was positioned directly above the center of the RF coil. Twelve CPMG echoes with a $300-\mu$ s echo spacing were measured in $5 \mathrm{~min}$; the summed echo displayed in the frequency domain clearly revealed the layered structure of the samples (Fig. 6A and B). The average frequency difference between adjacent peaks of the profiles (Fig. 6A and B) was about $110 \mathrm{kHz}$, meaning that the strength of the static gradient is about $9.9 \mathrm{~T} / \mathrm{m}$. This value is close to the simulation result of $10.2 \mathrm{~T} / \mathrm{m}$ (Fig. 3E). The static gradient strength experienced by the small latex sheet between $0.5 \mathrm{~mm}$ and $2.1 \mathrm{~mm}$ above the center of the RF coil

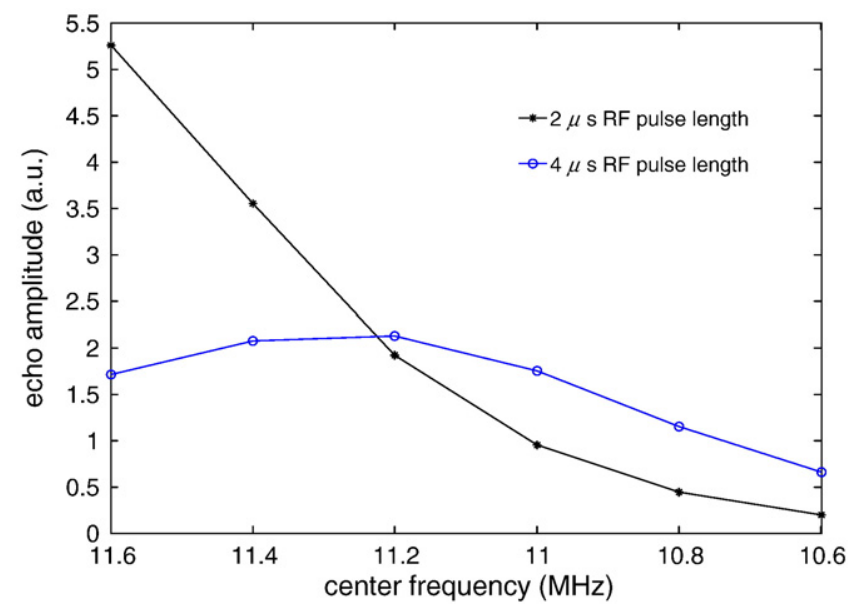

Fig. 5. The Hahn echo amplitude of the pencil eraser obtained at different center frequencies or detection depths with an RF pulse length of 2 or $4 \mu \mathrm{s}$. Experimental parameters were center frequency of $11.6-10.6 \mathrm{MHz}$, echo time of $210 \mu \mathrm{s}$, relaxation delay of $0.1 \mathrm{~s}$ and 600 averages for each point. The lowest center frequency of $10.6 \mathrm{MHz}$ corresponds to a vertical distance of about $3 \mathrm{~mm}$ away from the RF coil surface.

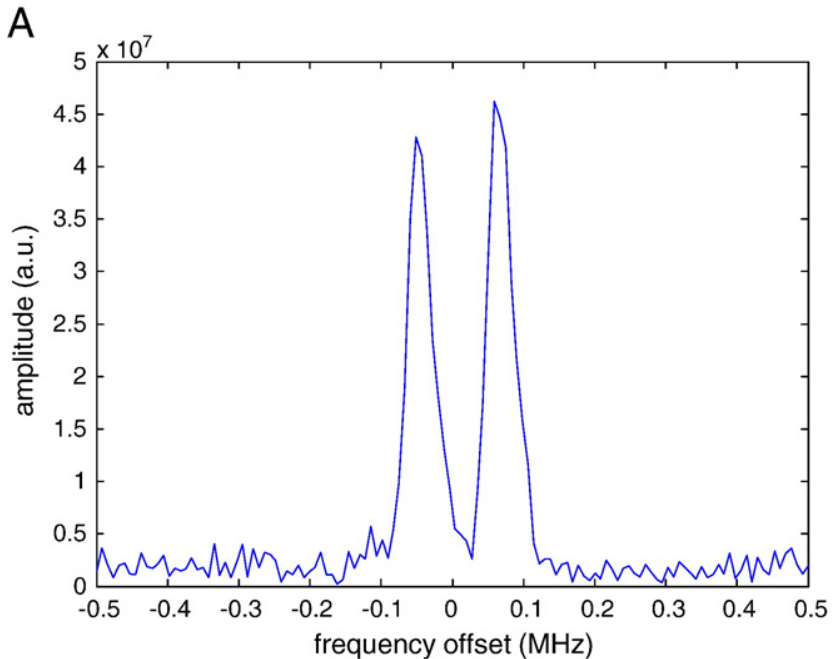

B

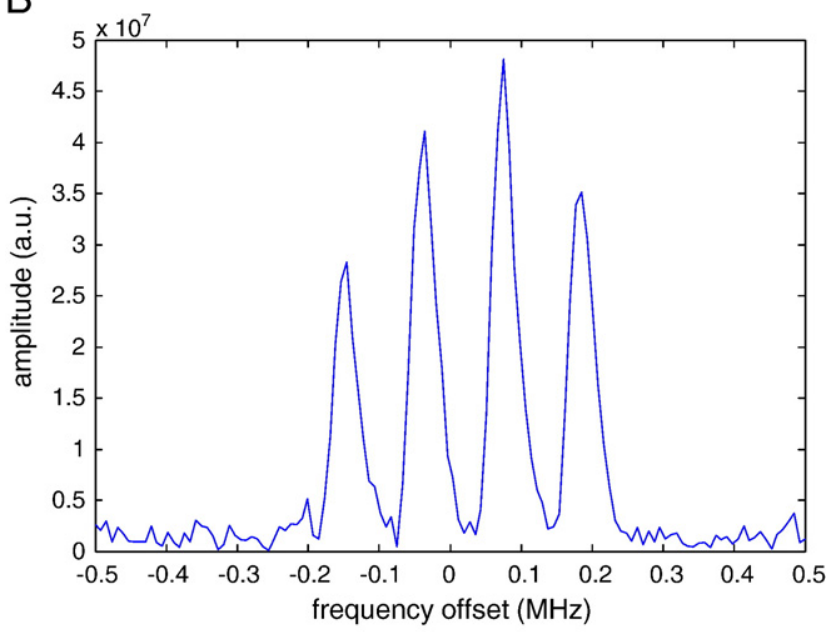

Fig. 6. (A) A one-dimensional image of the sample consisting of two small latex sheets separated by a transparency film. Experimental parameters not mentioned in the text were center frequency of $11.62 \mathrm{MHz}$, RF pulse length of $2 \mu$ s, relaxation delay of $0.1 \mathrm{~s}$ and 3000 averages. (B) A one-dimensional image of the sample containing four small latex sheets separated by transparency films. Experimental parameters were the same as those in Fig. $6 \mathrm{~A}$, except that the center frequency was $11.50 \mathrm{MHz}$. In both figures, the peak amplitudes were affected by distances between the latex sheets and RF coil, as well as the extent of off-resonance.

surface was further determined with increased precision by measuring the peak frequency of the latex sheet profile at 12 vertical positions and a least squares fit. The obtained static gradient strength was $10.23 \mathrm{~T} / \mathrm{m}$, which is quite close to the simulation result (Fig. 3E). The correlation coefficient of the fit was greater than 0.999 , implying that the static gradient strength $\left|\Delta \mathrm{B}_{0} / \Delta y\right|$ is approximately constant in the measured range and consistent with the simulation result (Fig. 3E). Additionally, the spatial resolution achieved using the $5 \times 5$ $\mathrm{mm}$ horizontal area was roughly estimated by subtracting the ideal spectral width spanned by the thickness of the latex sheet from the actual spectral width at the base of the latex sheet profile; the difference was about $85 \mathrm{kHz}$, corresponding to a spatial resolution of about $200 \mu \mathrm{m}$. 
Table 1

Self-diffusion coefficients of some liquids measured using the Hahn echo sequence [30] at $25^{\circ} \mathrm{C}$ and the corresponding values in literature (Bruker Almanac 2005)

\begin{tabular}{llll}
\hline & $\begin{array}{l}\mathrm{D} \text { (measured) } \\
\left(10^{-9} \mathrm{~m}^{2} \mathrm{~s}^{-1}\right)\end{array}$ & $\begin{array}{l}\mathrm{D} \text { (literature) } \\
\left(10^{-9} \mathrm{~m}^{2} \mathrm{~s}^{-1}\right)\end{array}$ & Error (\%) \\
\hline Formamide & 0.547 & 0.55 & 0.6 \\
Ethanol & 1.063 & 1.08 & 1.6 \\
$n$-Hexane & 4.097 & 4.26 & 3.8 \\
\hline
\end{tabular}

Experimental parameters for each data point were center frequency of 11.3 $\mathrm{MHz}, \mathrm{RF}$ pulse length of $4 \mu \mathrm{s}$, relaxation delay of $5 \mathrm{~s}$ and 24 averages.

\subsection{Liquid self-diffusion coefficient measurements}

The relatively uniform static gradient strength $\left|\Delta \mathrm{B}_{0} / \Delta \mathrm{y}\right|$ above the center of the magnet surface was also utilized to measure the self-diffusion coefficients of several liquids. The liquid investigated was placed in a small round bottle with an inner diameter of $0.85 \mathrm{~mm}$; the bottle center was positioned directly above the center of the RF coil. The Hahn echo sequence with CPMG echo-addition [30] was used for determining the self-diffusion coefficient of the liquid. For the low-viscosity liquid, the diffusive attenuation of the Hahn echo amplitude of the liquid placed in a static gradient is described by

$\ln \left(I / I_{0}\right) \approx-\frac{2}{3} \gamma^{2} G^{2} \tau^{3} D$

where $\gamma$ is the gyromagnetic ratio, $G$ is the static gradient strength, $\tau$ is the time spacing between $\theta_{x}$ and $2 \theta_{y}$ RF pulses, $D$ is the self-diffusion coefficient of the liquid, $I$ is the Hahn echo amplitude at echo time $2 \tau$, and $I_{0}$ is the Hahn echo amplitude at the shortest echo time in the experiment [30]. First, deionized water $\left(D=2.299 \times 10^{-9} \mathrm{~m}^{2} / \mathrm{s}\right.$ at $25^{\circ} \mathrm{C}$, Bruker Almanac 2005) was placed in the bottle and used to determine the static gradient strength experienced by the excited volume. 1000 CPMG echoes with a $40-\mu$ s echo spacing were generated after the Hahn echo, and all echoes were summed to increase the SNR [30]. Twelve different $\tau$ values were used; the experimental time for each $\tau$ value was $2 \mathrm{~min}$. The static gradient strength experienced by water in the excited volume was extracted from the term $-2 / 3 \gamma^{2} G^{2} D$ in Eq. (1) using the least squares fitting. The measurement procedure was conducted three times; average static gradient strength obtained from the three measurements was $10.39 \mathrm{~T} / \mathrm{m}$. This value was slightly larger than $10.23 \mathrm{~T} / \mathrm{m}$ obtained in Section 3.2 ; this difference can likely be ascribed to the larger crosssection of the round bottle compared with that of the small latex sheet $(5 \times 5 \mathrm{~mm})$, and a relatively large horizontal area above the center of the magnet surface experiences a strong static gradient strength (Fig. 2). Moreover, the standard deviation of the three measurements was $1.2 \%$, implying that the measurement has sufficient precision.

Using water as the reference, the self-diffusion coefficients of ethanol (99.5\%; Shimakyu, Niigata, Japan), $n$ hexane (99.1\%; Mallinckrodt Baker, Phillipsburg, NJ, USA) and formamide (99.5\%; Merck Chemicals, Darmstadt, Germany) were determined in turn by the aforementioned process. Table 1 lists the obtained self-diffusion coefficients of the liquids. The measured values are close to those in literature (Bruker Almanac 2005), indicating that the proposed apparatus can be employed to determine selfdiffusion coefficients of liquids with small errors.

\subsection{Applications of the apparatus}

Three applications were conducted to demonstrate the efficiency of the proposed single-sided mobile NMR apparatus for non-destructive material inspection; similar applications have also been reported elsewhere [2,3,22,23,31-33]. The first application is to differentiate between high-density polyethylene (HDPE) grains and low-density polyethylene (LDPE) grains (Formosa Plastics, Taipei, Taiwan) by measuring their respective $\mathrm{T}_{2}$ relaxation decays using the Hahn echo sequence $[2,3,22]$. During each measurement, the PE grains were simply placed on the RF coil. Results showed that the HDPE grains relaxed significantly faster than their LDPE counterparts (Fig. 7); therefore, the proposed apparatus can be used to distinguish between the HDPE and LDPE grains without ambiguity.

The second application is to monitor the hardening process of epoxy putty by continuously measuring the effective $\mathrm{T}_{2}$ relaxation time using the CPMG pulse sequence $[2,3,23]$. The samples comprised regular-set and fast-set epoxy putties obtained from a local manufacturer. The nominal set time for the regular-set and fast-set epoxy putties were 90 and 5 min, respectively. Each epoxy putty was mixed and pasted onto a cover glass. The cover glass

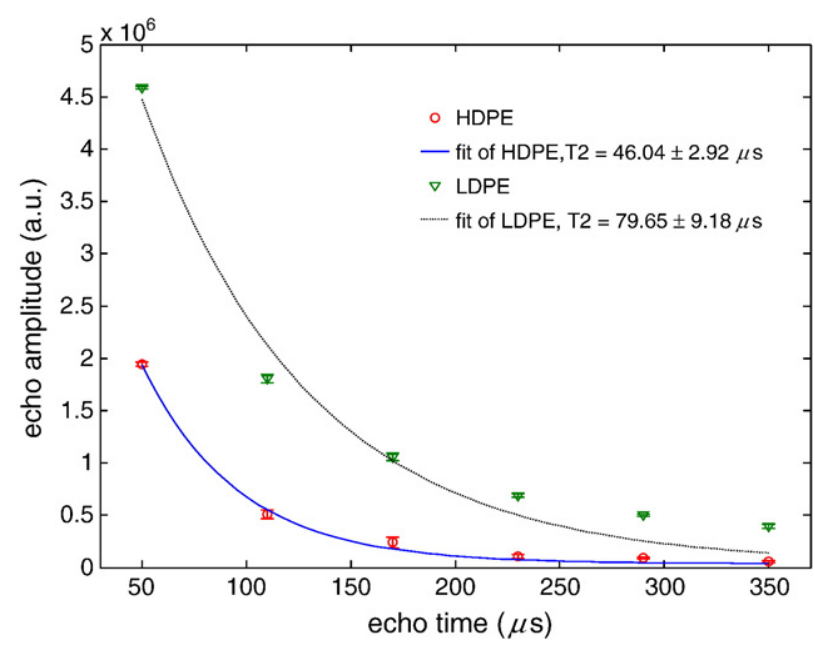

Fig. 7. The $T_{2}$ relaxation decays of the HDPE and the LDPE grains and their fit curves. Experimental parameters were center frequency of 11.4 $\mathrm{MHz}, \mathrm{RF}$ pulse length of $4 \mu \mathrm{s}$, relaxation delay of $0.1 \mathrm{~s}$, and 3000 averages for each measurement. The measurements were conducted three times; the standard deviations of three measurements are represented by error bars. Using a single exponential decay model and a least squares fit, the $\mathrm{T}_{2}$ relaxation times of HDPE and LDPE grains were found to be $46.04 \pm 2.92$ and $79.65 \pm 9.18 \mu$ s, respectively. 
was then placed on the RF coil; 500 CPMG echoes with an echo spacing of $40 \mu \mathrm{s}$ were acquired in $5 \mathrm{~min}$. A single exponential decay model was used to fit the CPMG echo train and then obtain the effective $T_{2}$ relaxation time of the epoxy putty. A 1-min idle time was inserted between adjacent measurements to decrease the hardware duty cycle, and measurements were repeated for $6 \mathrm{~h}$ for each epoxy putty sample. The effective $T_{2}$ relaxation times of both epoxy putties showed a fast initial decrease and then decayed slowly (Fig. 8). Since the effective $T_{2}$ relaxation times measured in inhomogeneous $\mathrm{B}_{0}$ and $\mathrm{B}_{1}$ magnetic fields are dependent on echo spacing [34], the measured turning points of the effective $\mathrm{T}_{2}$ curves may not agree with set times specified by the manufacturer. Nevertheless, experiments clearly revealed that the fast-set epoxy putty reached the turning point of the effective $T_{2}$ curve earlier than the regular-set epoxy putty, and late in the setting process, the hydrogen nuclei in the regular-set epoxy putty became less mobile than those in the fast-set epoxy putty (Fig. 8). Therefore, the hardening process of the epoxy putties can be characterized by the proposed apparatus, at least qualitatively.

The third application correlates fat content in milk powder with its CPMG echo amplitude [31-33]. Different amounts of whole milk powder (fat content $=28.2 \%$; Nestle Taiwan, Taipei, Taiwan) and nonfat milk powder (fat content $=1.7 \%$; Nestle Taiwan) were blended into $5 \mathrm{~g}$ of mixed powders with several intermediate fat percentages. The mixed milk powders were sealed in small plastic bags. The fat content percentages of whole milk powder and non-fat milk powder were obtained from product labels. Each mixed milk powder bag was placed on the RF coil; the volume of each packet was large enough to fill the entire sensitive volume of the apparatus. For each packet, four CPMG echoes with an echo spacing of $1 \mathrm{~ms}$ were acquired and added together to increase

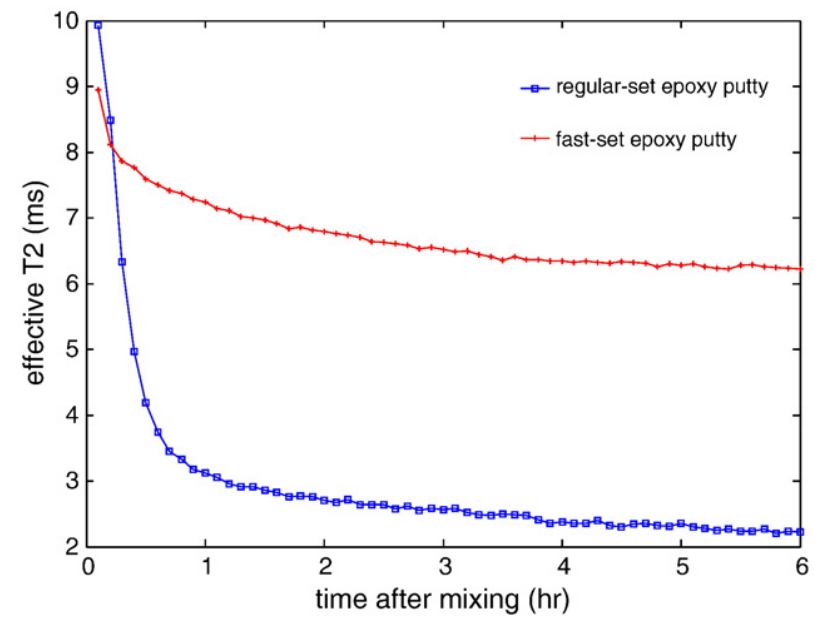

Fig. 8. The effective $\mathrm{T}_{2}$ relaxation times of the epoxy putties versus the time length after mixing. Experimental parameters not mentioned in the text were center frequency of $11.4 \mathrm{MHz}, \mathrm{RF}$ pulse length of $4 \mu \mathrm{s}$, relaxation delay of $0.1 \mathrm{~s}$ and 3000 averages for each measurement.

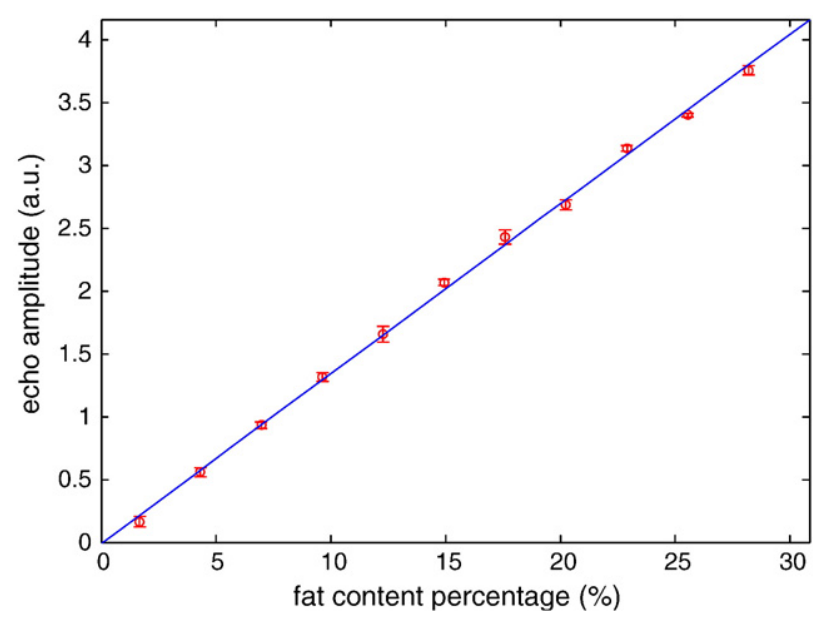

Fig. 9. Average CPMG echo amplitudes of the mixed milk powder packets versus fat content percentages, and the fit line. The error bars represent standard deviations of the three measurements. Experimental parameters not mentioned in the text were center frequency of $11.4 \mathrm{MHz}$, RF pulse length of $4 \mu \mathrm{s}$, relaxation delay of $0.2 \mathrm{~s}$ and 1500 averages for each measurement.

the SNR. The plastic bag was only $0.04 \mathrm{~mm}$ thick and generated no observable signal when measured alone. Moreover, the water content in mixed milk powders, calculated from the product labels, was $<5 \%$. Signals from these small amounts of water were further attenuated by the static gradient of the apparatus and the $1 \mathrm{~ms}$ echo spacing chosen. Consequently, the small water content in the mixed milk powders should not interfere with the measurement results $[32,33]$. Measurement time for each packet was $5 \mathrm{~min}$; measurements were conducted three times. Average echo amplitudes of the mixed milk powder packets were plotted versus the corresponding fat content percentages and fitted by a straight line using the least squares method (Fig. 9). The echo amplitudes are strongly correlated with the fat percentages of the mixed milk powders (correlation coefficient $>0.99$ ), implying that the proposed apparatus can be employed to estimate the fat content of milk powders once a suitable calibration line is established.

\section{Discussion}

During the one-dimensional imaging experiments or liquid self-diffusion coefficient measurements, samples had to be restricted to the region directly above the RF coil center, where the contour lines of the $\mathrm{B}_{0}$ field strength are relatively flat, and consequently, only part of the RF coil area was utilized. In this case, the original RF coil can be replaced with a smaller RF coil that only covers the area utilized [10]. In this way, the filling factor of the RF coil will increase and coil resistance decrease, probably increasing the SNR. Furthermore, the sample can therefore have a cross-section that is larger than the area of the modified RF coil because the RF coil is only sensitive to the space directly above. Moreover, the $\mathrm{B}_{0}$ strength contour lines above the apparatus may be 
adjusted by separating the magnet along its magnetization direction [10] or adding shim magnets above the magnet $[20,21]$. In this manner, more uniform static gradient strength or better $\mathrm{B}_{0}$ homogeneity above the apparatus may be achieved compared with the present design. Implementing these modifications will be our future work.

\section{Conclusions}

A mobile, single-sided NMR apparatus was realized simply by using a single, disc-shaped permanent magnet polarized in radial direction. The simple design reduces the effort and cost required for apparatus construction; furthermore, the $\mathrm{B}_{0}$ magnetic field generated is parallel to the apparatus surface, allowing relatively flexible selection of the RF coil for the apparatus. The proposed apparatus can acquire a clear Hahn echo of a pencil eraser block lying on the RF coil in one shot and therefore has sufficient sensitivity. The static magnetic field gradient above the center of the apparatus surface enables the apparatus to conduct one-dimensional imaging of thin samples and measure self-diffusion coefficients of some liquids with small errors. Several material inspection experiments also demonstrate the potential of the proposed apparatus for nondestructive testing. Modifying the present magnet structure to enhance the performance of the apparatus will be the future work.

\section{Acknowledgments}

We thank the National Science Council of the Republic of China, Taiwan, for financially supporting this research under Contract No. NSC 96-2752-M-002-017-PAE, NSC 972752-M-002-010-PAE and NSC 96-2113-M-001-020. The National Center for High-Performance Computing in Taiwan is appreciated for providing the Maxwell3D software. Formosa Plastics of Taiwan is also commended for providing the HDPE and LDPE grain samples, as is Mr. Chau-Yi Chung from Academia Sinica, Taiwan, for manufacturing the magnet holder. Ted Knoy is appreciated for his editorial assistance.

\section{References}

[1] Blümich B. NMR imaging of materials. Oxford: Clarendon Press; 2000.

[2] Blümich B. Essential NMR. Berlin: Springer-Verlag; 2005.

[3] Blümich B, Perlo J, Casanova F. Mobile single-sided NMR. Prog Nucl Magn Reson Spectrosc 2008;52:197-269.

[4] Eidmann G, Savelsberg R, Blümler P, Blümich B. The NMR MOUSE, a mobile universal surface explorer. J Magn Reson A 1996;122:104-9.

[5] Blümich B, Anferov V, Anferova S, Klein M, Fechete R, Adams M, et al. Simple NMR-MOUSE with a bar magnet. Concept Magn Reson B 2002;15:255-61.

[6] Le Bec G, Yonnet JP, Raoof K. Coil and magnet design for nuclear magnetic resonance in inhomogeneous field. IEEE Trans Magn 2006; 42:3861-7.
[7] Chang WH, Chen JH, Hwang LP. Single-sided mobile NMR with a Halbach magnet. Magn Reson Imaging 2006;24:1095-102.

[8] Marble AE, Mastikhin IV, Colpitts BG, Balcom BJ. A constant gradient unilateral magnet for near-surface MRI profiling. J Magn Reson 2006;183:228-34.

[9] McDonald PJ, Aptaker PS, Mitchell J, Mulheron M. A unilateral NMR magnet for sub-structure analysis in the built environment: the Surface GARField. J Magn Reson 2007;185:1-11.

[10] Perlo J, Casanova F, Blümich B. Profiles with microscopic resolution by single-sided NMR. J Magn Reson 2005;176:64-70.

[11] Rahmatallah S, Li Y, Seton HC, Mackenzie IS, Gregory JS, Aspden RM. NMR detection and one-dimensional imaging using the inhomogeneous magnetic field of a portable single-sided magnet. J Magn Reson 2005;173:23-8.

[12] Prado PJ. Single sided imaging sensor. Magn Reson Imaging 2003;21: 397-400.

[13] Bray CL, Hornak JP. Unilateral MRI using a rastered projection. J Magn Reson 2007;188:151-9.

[14] Perlo J, Casanova F. Blümich B. 3D imaging with a single-sided sensor: an open tomograph. J Magn Reson 2004;166:228-35.

[15] Fukushima E, Jackson JA. Unilateral magnet having a remote uniform field region for nuclear magnetic resonance. US Patent $6,489,872$.

[16] Kato H, Kishi K, Takahashi N, Asaumi JI, Honda Y, Yanagi Y, et al. A design of permanent magnet array for unilateral NMR device. Concept Magn Reson B 2008;33B:201-8.

[17] Marble AE, Mastikhin IV, Colpitts BG, Balcom BJ. A compact permanent magnet array with a remote homogeneous field. J Magn Reson 2007;186:100-4.

[18] Manz B, Coy A, Dykstra R, Eccles CD, Hunter MW, Parkinson BJ, et al. A mobile one-sided NMR sensor with a homogeneous magnetic field: the NMR-MOLE. J Magn Reson 2006;183:25-31.

[19] Marble AE, Mastikhin IV, Colpitts BG, Balcom BJ. An analytical methodology for magnetic field control in unilateral NMR. J Magn Reson 2005;174:78-87.

[20] Perlo J, Casanova F, Blümich B. Ex situ NMR in highly homogeneous fields: H-1 spectroscopy. Science 2007;315:1110-2.

[21] Perlo J, Demas V, Casanova F, Meriles CA, Reimer J, Pines A, et al. High-resolution NMR spectroscopy with a portable single-sided sensor. Science 2005;308:1279.

[22] Blümich B, Casanova F, Perlo J, Anferova S, Anferov V, Kremer K, et al. Advances of unilateral mobile NMR in nondestructive materials testing. Magn Reson Imaging 2005;23:197-201.

[23] Blümich B, Anferova S, Kremer K, Sharma S, Herrmann V, Segre A. Unilateral nuclear magnetic resonance for quality control - The NMRmouse. Spectroscopy 2003;18:18-32.

[24] Guthausen G, Guthausen A, Balibanu F, Eymael R, Hailu K, Schmitz U, et al. Soft-matter analysis by the NMR-MOUSE. Macromol Mater Eng 2000;276:25-37.

[25] Proietti N, Capitani D, Rossi E, Cozzolino S, Segre AL. Unilateral NMR study of a XVI century wall painted. J Magn Reson 2007;186: 311-8.

[26] Blümich B, Anferova S, Sharma S, Segre AL, Federici C. Degradation of historical paper: nondestructive analysis by the NMR-MOUSE. J Magn Reson 2003;161:204-9.

[27] Brai M, Camaiti M, Casieri C, De Luca F, Fantazzini P. Nuclear magnetic resonance for cultural heritage. Magn Reson Imaging 2007; 25:461-5.

[28] Presciutti F, Perlo J, Casanova F, Glöggler S, Miliani C, Blümich B, et al. Noninvasive nuclear magnetic resonance profiling of painting layers. Appl Phys Lett 2008;93:033505.

[29] Anferova S, Anferov V, Adams M, Blümler P, Routley N, Hailu K, et al. Construction of a NMR-MOUSE with short dead time. Concept Magn Reson B 2002;15:15-25.

[30] Rata DG, Casanova F, Perlo J, Demco DE, Blümich B. Self-diffusion measurements by a mobile single-sided NMR sensor with improved magnetic field gradient. J Magn Reson 2006;180:229-35. 
[31] Petrov OV, Hay J, Mastikhin IV, Balcom BJ. Fat and moisture content determination with unilateral NMR. Food Res Int 2008;41: 758-64.

[32] Veliyulin E, Mastikhin IV, Marble AE, Balcom BJ. Rapid determination of the fat content in packaged dairy products by unilateral NMR. J Sci Food Agric 2008;88:2563-7.
[33] Guthausen A, Guthausen G, Kamlowski A, Todt H, Burk W, Schmalbein D. Measurement of fat content of food with single-sided NMR. J Am Oil Chem Soc 2004;81:727-31.

[34] Guthausen A, Zimmer G, Blümler P, Blümich B. Analysis of polymer materials by surface NMR via the MOUSE. J Magn Reson 1998;130: $1-7$. 